\title{
Midinfrared InAsSbN/InAs Multiquantum Well Light-Emitting Diodes
}

\author{
P. J. Carrington, M. de la Mare, K. J. Cheetham, Q. Zhuang, and A. Krier \\ Department of Physics, Lancaster University, Lancaster LA1 4YB, UK \\ Correspondence should be addressed to P. J. Carrington, p.carrington@lancaster.ac.uk
}

Received 23 April 2011; Revised 10 June 2011; Accepted 5 July 2011

Academic Editor: Yuh-Jen Cheng

Copyright () 2011 P. J. Carrington et al. This is an open access article distributed under the Creative Commons Attribution License, which permits unrestricted use, distribution, and reproduction in any medium, provided the original work is properly cited.

Electroluminescence is reported from dilute nitride InAsSbN/InAs multiquantum well light-emitting diodes grown using nitrogen plasma source molecular beam epitaxy. The diodes exhibited bright emission in the midinfrared peaking at $3.56 \mu \mathrm{m}$ at room temperature. Emission occurred from a type I transition from electrons in the InAsSbN to confined heavy and light hole states in the QW. Analysis of the temperature- and current-dependent electroluminescence shows that thermally activated hole leakage and Auger recombination are the performance limiting factors in these devices.

\section{Introduction}

There is increasing interest in the fabrication of midinfrared optoelectronic devices for a range of applications including environmental pollution monitoring, remote sensing (LIDAR), infrared counter-measures, and free space optical communications. A number of different semiconductor materials and devices are currently being investigated including InSb/InAs quantum dots [1], bulk GaInAsSbP pentanary [2] and AlInSb alloys [3], InGaAsSb/AlGaInAsSb quantum wells (QWs) [4], and InAs/GaSb superlattices [5]. Recently we reported on bright room temperature electroluminescence from type II InAsSb/InAs multiquantum well (MQW) light-emitting diodes (LEDs) which produced a quasi-cw power of $12 \mu \mathrm{W}$ corresponding to an internal quantum efficiency of $2.2 \%$ at a peak wavelength near $3.7 \mu \mathrm{m}$ [6]. One way to improve the performance is to introduce nitrogen into the well. The introduction of a small amount of nitrogen reduces the bandgap without excess strain and can change the band alignment from type II to type I increasing the electron confinement and e-h wavefunction overlap. Furthermore dilute nitrides have the potential to reduce Auger recombination [7] and intervalence band absorption.

To date there have been no reports of LEDs based on dilute nitride material in the mid-infrared spectral range $(\lambda>$ $3 \mu \mathrm{m}$ ) due to the difficulty in growing epitaxial material of sufficient quality. Our previous work has concentrated on the development and optimisation of the growth of InAsSbN and from which photoluminescence (PL) was obtained up to $250 \mathrm{~K}$ from InAsSbN/InAs MQWs $[8,9]$. It was found that the surfactant effect of $\mathrm{Sb}$ enhances the crystalline quality and PL intensity. Also the possibility to adjust the Sb and $\mathrm{N}$ contents in the material enables one to grow the material lattice matched to InAs or to tailor the strain in the QW. In this work we report bright room temperature electroluminescence at $3.56 \mu \mathrm{m}$ from InAsSbN/InAs MQW LEDs and investigate the temperature and current dependencies of the confined transitions in the QWs.

\section{Sample Growth and Fabrication}

The LED (Figure 1(a)) was grown by solid source molecular beam epitaxy (MBE) on (001) InAs substrates using a radio frequency plasma nitrogen source. Thermal effusion K-cells were used to supply the group III fluxes and two Veeco valved cracker cells were used to provide the antimony and arsenic fluxes. A $0.5 \mu \mathrm{m}$ n-type InAs(Te) layer was grown first at $490^{\circ} \mathrm{C}$ at $1 \mu \mathrm{m} / \mathrm{h}$, followed by the MQW active region which was grown at $410^{\circ} \mathrm{C}$, with a growth rate of $0.5 \mu \mathrm{m} / \mathrm{h}$. The thicknesses of the well and barrier layers were 10 and $25 \mathrm{~nm}$, respectively. An undoped $30 \mathrm{~nm} \mathrm{Al}_{0.9} \mathrm{Ga}_{0.1} \mathrm{As}_{0.21} \mathrm{Sb}_{0.79}$ electron blocking barrier was grown $100 \mathrm{~nm}$ above the active region and finally a $0.5 \mu \mathrm{m}$ p-type $\operatorname{InAs}(\mathrm{Be})$ layer was grown at $490^{\circ} \mathrm{C}$. The QWs were grown using the same shutter 


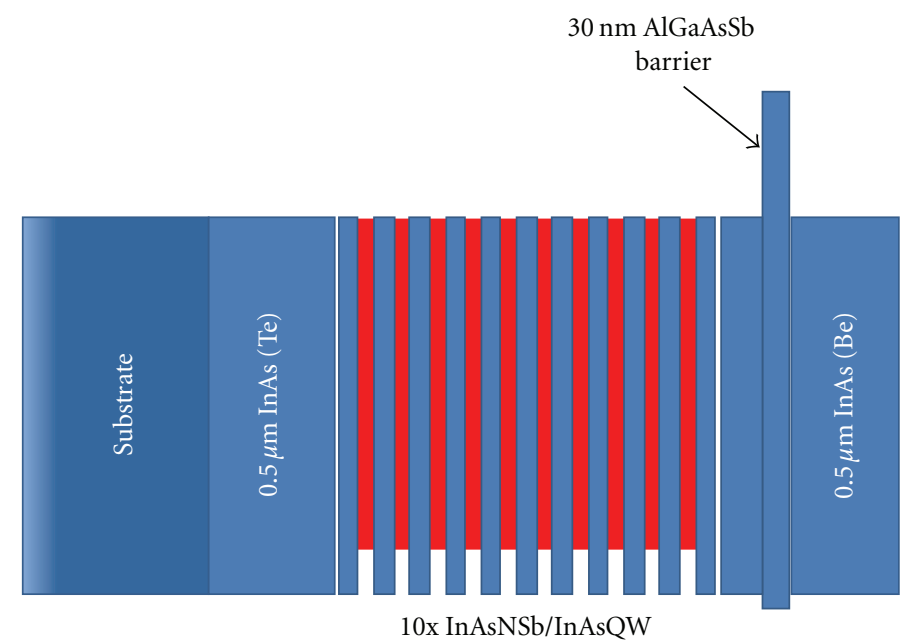

(a)

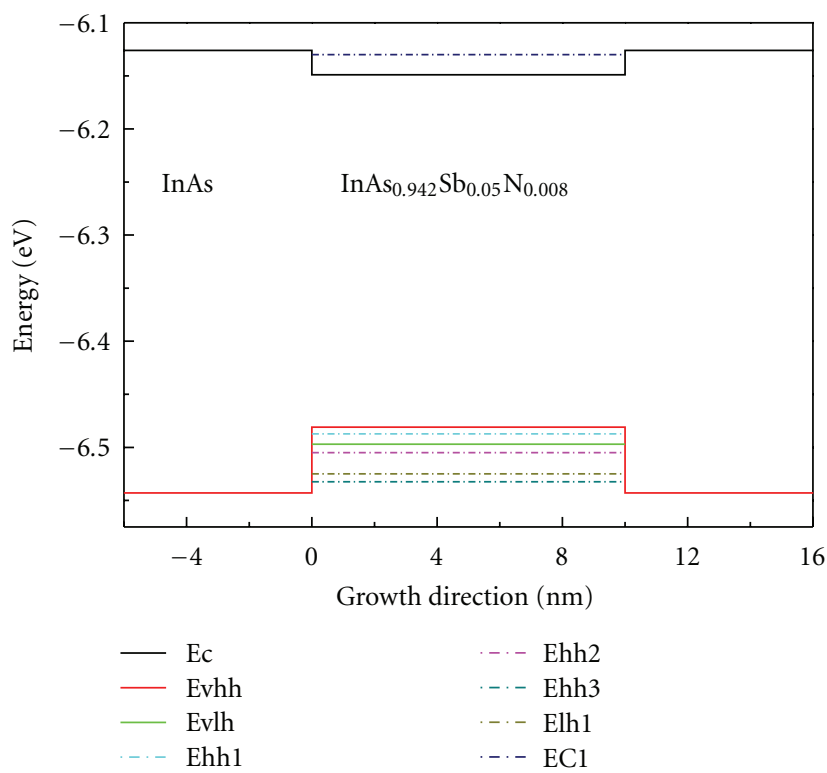

\begin{tabular}{|c|c|}
\hline Transition & Energy $(\mathrm{eV})$ \\
\hline Ec-Ehh1 & 0.357 \\
\hline Ec-Elh1 & 0.395 \\
\hline Ehh1-InAs & 0.056 \\
\hline Elh1-InAs & 0.018 \\
\hline Ec-InAs & 0.004 \\
\hline
\end{tabular}

(b)

Figure 1: (a) Schematic diagram of the InAs $\mathrm{s}_{0.942} \mathrm{Sb}_{0.05} \mathrm{~N}_{0.008} /$ InAs MQW LED structure. (b) The calculated $4 \mathrm{~K}$ (flat) band structure. The table lists the transitions from the conduction band to the heavy and light hole ground states, Ec-Ehh1 and Ec-Elh1, Ehh1-InAs, and so forth correspond to the carrier localization energies.

sequence as described previously [6] to improve the interface abruptness. For the growth of the QWs the nitrogen plasma setting was fixed at a power of $210 \mathrm{~W}$ and a nitrogen flux of $7.0 \times 10^{-7} \mathrm{mbar}$, whilst the $\mathrm{Sb}$ and As fluxes were set at $1.1 \times 10^{-6}$ and $2.6 \times 10^{-6} \mathrm{mbar}$, respectively. During the QW growth the RHEED pattern showed a clear $1 \times 3$ pattern which quickly changed to $2 \times 4$ under the $\mathrm{As}_{2}$ soak prior to the growth of the barrier. High-resolution double crystal X-ray diffraction (DCXRD) was used to determine the $\mathrm{N}$ and $\mathrm{Sb}$ compositions. The resulting wafers were then processed into $1 \mathrm{~mm}$ diameter mesa-etched LEDs using standard photolithographic techniques and mounted on TO46 headers. The devices were tested at $1 \mathrm{kHz}$ using a $50 \%$ duty cycle and the electroluminescence (EL) was measured using a Bentham $0.3 \mathrm{~m}$ monochromator and $77 \mathrm{~K}$ InSb photodiode.
Output power at room temperature was measured using an integrated sphere and calibrated $\mathrm{PbSe}$ photodetector.

\section{Results and Discussion}

Figure 2 shows the X-ray diffraction spectrum measured from one of the LEDs which shows a number of welldefined satellite peaks, where the FWHM of the satellite peaks is around 80 arcsecs characteristic of a high structural quality. This is slightly higher than previous InAsSb/InAs MQWs [6] indicating that some minor structural disorder has been introduced by the nitrogen. The broader peak around 1000 arcsec is from the AlGaAsSb barrier (which was nonintentionally grown slightly lattice mismatched). 


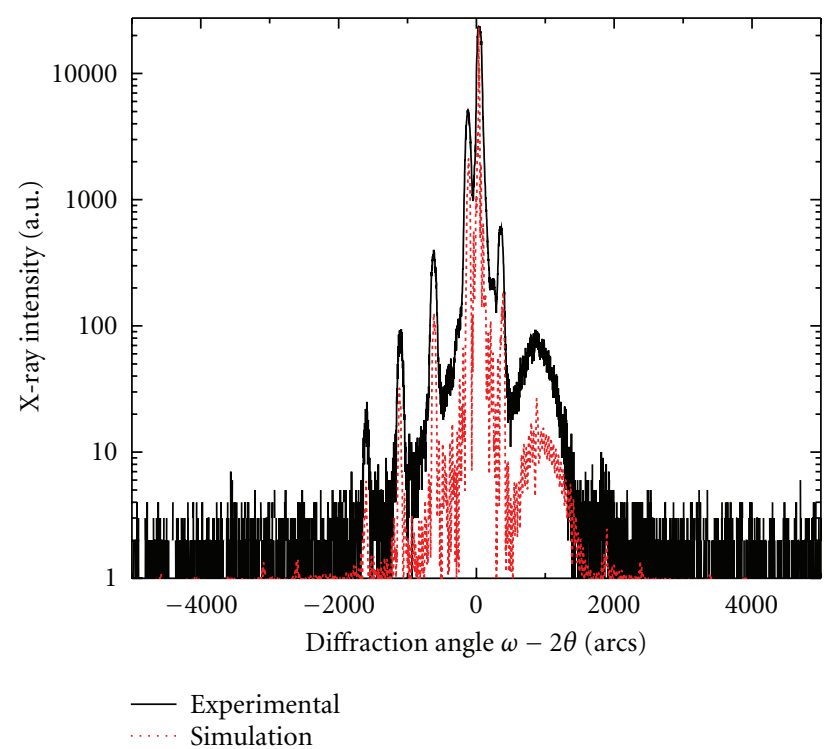

Figure 2: High-resolution X-ray diffraction spectrum of the InAsSbN/InAs LED. Solid line (black): experimental results, dashed line (red): simulation.

Simulation of the XRD curve gives the $\mathrm{Sb}$ and $\mathrm{N}$ contents in the well as $5.0 \%$ and $0.8 \%$, respectively, so that the strain in the QW is approximately $0.2 \%$. The band structure at $4 \mathrm{~K}$ for the QW is shown in Figure 1(b) using the same calculation procedure as described previously [9]. Three heavy hole and one light hole states are confined. The transition energies and localization energies are given in the adjacent table. As the e 1 localisation energy is negligible $\sim 4 \mathrm{meV}$, one can ignore the electron confinement in the QW.

The temperature-dependent current-voltage $(I-V)$ curves measured from one of the LEDs are shown in Figure 3. The series resistance was calculated to be $\sim 2.5 \Omega$ and was found to be independent of temperature. The leakage current in narrow gap semiconductors is much more problematic than at shorter wavelengths due to the higher intrinsic carrier concentration and intrinsic point defects $[10]$ and this is reflected in the device $I-V$ characteristics. In this case the diode leakage current was found to be $2 \mathrm{~mA}$ at $0.5 \mathrm{~V}$ at room temperature which is lower than InAs-based devices made previously in our laboratory [11] and comparable to those reported in [12]. With decreasing temperature the leakage current decreases to $8 \times 10^{-4} \mathrm{~A}$ at $0.5 \mathrm{~V}$ (at $4 \mathrm{~K}$ ) and is almost temperature independent over the $4-150 \mathrm{~K}$ range suggesting that the process responsible for leakage could be due to tunnelling effects. At low temperatures the occupation of traps is higher meaning that the forward and reverse currents at $0.5 \mathrm{~V}$ are similar in magnitude. Reduction in surface states to reduce the leakage current and to improve device performance can be expected using surface passivation as demonstrated previously [13]. At room temperature the zero bias differential resistance area product $\left(R_{0} A\right)$ was determined to be $1.7 \Omega \cdot \mathrm{cm}^{2}$. Analysis of the slope near the origin gives a value of 2 for the ideality

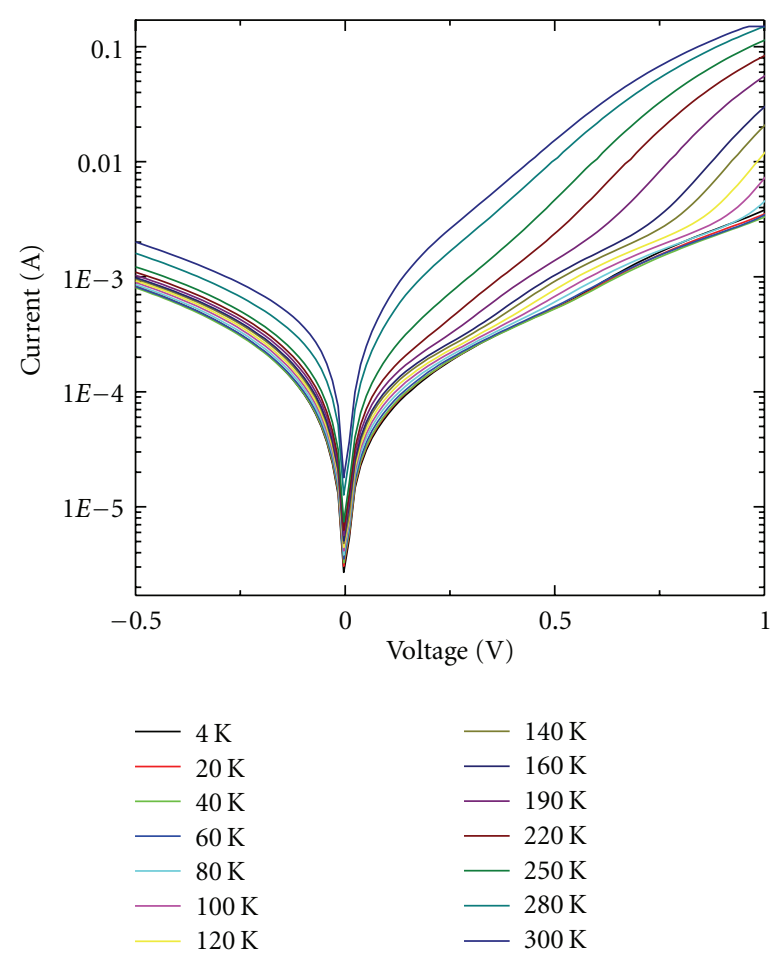

FIgURE 3: Semilogarithmic plot of the temperature-dependent current-voltage $(I-V)$ curves obtained from one of the InAsSbN QW LEDs.

factor indicating that generation recombination current dominates.

Low temperature $(4 \mathrm{~K})$ electroluminescence (EL) emission spectra measured from the LED at various currents are shown in Figure 4. At $30 \mathrm{~mA}$ a single symmetric peak is observed at $0.366 \mathrm{eV}$ with a FWHM of $11 \mathrm{meV}$. This agrees well with the value of $0.357 \mathrm{eV}$ calculated for the electronheavy hole (e-hh1) transition (Figure 1(b)). As the current increases there is a small blue shift $\sim 2 \mathrm{meV}$ of the peak position due to band filling and two high energy shoulders appear. The inset of Figure 4 shows the deconvolution of the spectrum at $150 \mathrm{~mA}$ into three Gaussian peaks where the peak emission energies are $0.368,0.384$, and $0.415 \mathrm{eV}$. The latter two peaks correspond to recombination from elh1 (calculated value of $0.395 \mathrm{eV}$ ) and InAs. The small blueshift of the QW emission peak ( $2 \mathrm{meV}$ ) compared to type II InAsSb/InAs $(\sim 8 \mathrm{meV})[6]$ suggests that the band alignment is type I. Furthermore, the type II InAsSb/InAs followed a $\Delta E \sim$ current $^{1 / 3}$ behaviour due to band bending at the type II interface whereas no such dependence was found for the InAsSbN/InAs QWs.

The temperature-dependent EL at an injection current of $100 \mathrm{~mA}$ is shown in Figure 5. Emission from e-hh1 in the QW is the main peak which is observed up to room temperature whereas, emission from e-lh1 is not observed above $150 \mathrm{~K}$. At high temperatures $(T>200 \mathrm{~K})$ a high energy tail is observed due to the Boltzmann thermal distribution of carriers. The temperature dependence of the peak energy is 


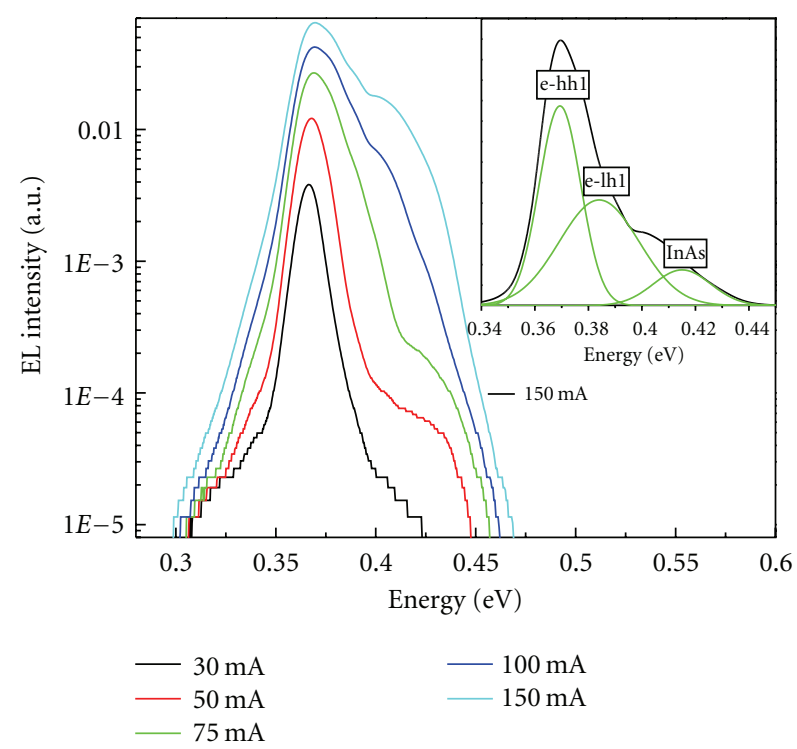

FIgURE 4: EL spectra measured at $4 \mathrm{~K}$ using increasing injection current at $1 \mathrm{kHz}, 50 \%$ duty cycle. The inset shows the de-convoluted spectra at $150 \mathrm{~mA}$ revealing three Gaussian peaks with peak emission energies which correspond to radiative recombination from e-hh1, e-lh1, and also InAs band-band recombination.

shown in Figure 6.The solid lines represent the fitting results using the empirical Varshni equation [14]

$$
E_{g}(T)=E_{g}(0)-\frac{\alpha T^{2}}{T+\beta},
$$

where $E_{g}(0), \alpha$, and $\beta$ are Varshni parameters and $T$ is the measured temperature. The values obtained for the Varshni fitting parameters are given in the table along with reference values for InAs and InAsN $[15,16]$. The temperature dependence of the InAsN bandgap is much less than that of InAs (lower value of $\alpha$ ) and the values for InAsSbN are slightly higher than InAsN. The carrier localization energy at a given temperature is given by [17]

$$
E_{\mathrm{loc}}(T)=E_{g}(T)-E_{\mathrm{PL}}(T),
$$

where $E_{\mathrm{PL}}(T)$ is the experimental $\mathrm{PL}$ peak. At $4 \mathrm{~K}$ the localization energy is $4 \mathrm{meV}$ which decreases to zero at $80 \mathrm{~K}$. The weak localization energy is related to the good structural quality and improvement in the nitrogen homogeneity produced by the surfactant effect of $S b$.

Figure 7(a) shows the temperature quenching for the ehh1 transition, normalized to 1 at $4 \mathrm{~K}$, where the integrated intensity drops by two orders of magnitude from $4-300 \mathrm{~K}$. Figure 7(b) is an Arrhenius plot of the same data from which an activation energy of $60 \mathrm{meV}$ was obtained from the high temperature region. This energy is attributed to the leakage of holes out of the QW (the energy is close to the calculated hh1 localization energy of $56 \mathrm{meV}$ ) and is considered to be the main reason for the quenching of the luminescence. As noted previously the lh1 transition is not observed above $150 \mathrm{~K}$ as the localization energy is only $18 \mathrm{meV}$ and holes can easily escape above this temperature.

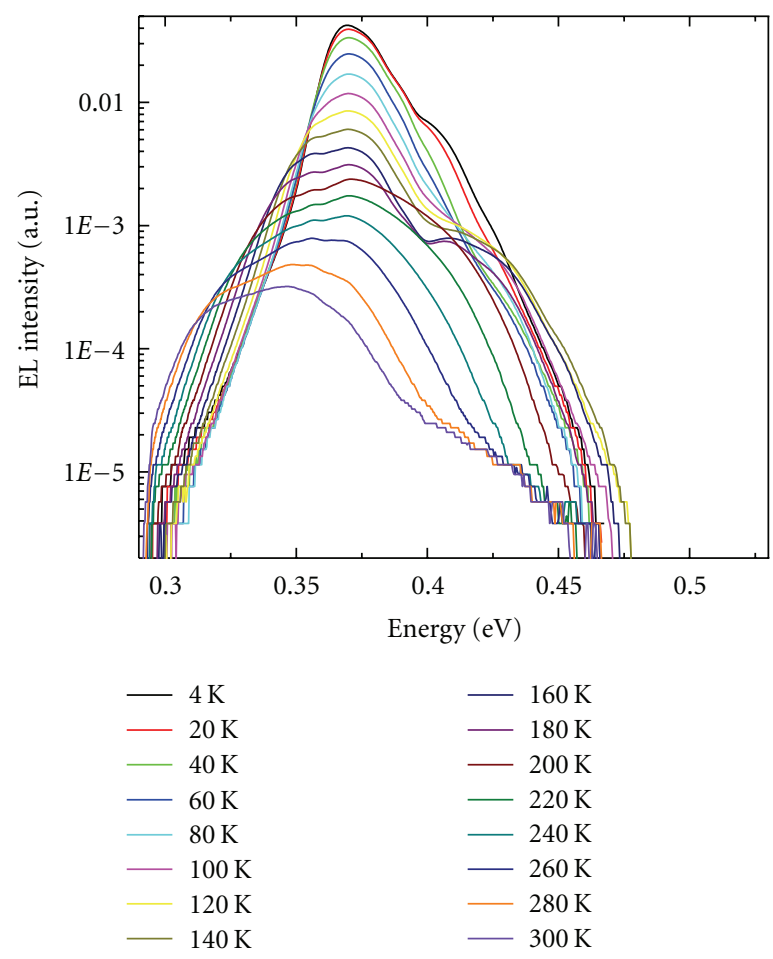

FIGURE 5: The temperature-dependent electroluminescence emission spectra measured using $100 \mathrm{~mA}$ and $1 \mathrm{kHz}, 50 \%$ duty cycle.

Figure 8(a) shows the room temperature LED emission spectra measured using different currents where the peak emission occurs at $0.345 \mathrm{eV}$ with a FWHM of $58 \mathrm{meV}$. (The slight asymmetry on the low energy side of the peak is due to hydrocarbon absorption contamination on the cryostat window.) LED output power (using $1 \mathrm{kHz}$ ac injection current at $50 \%$ duty cycle) was measured at room temperature using an integrating sphere and calibrated $\mathrm{PbSe}$ photodetector (Figure 8(b)). An output power of approximately $6 \mu \mathrm{W}$ was obtained at $100 \mathrm{~mA}$ corresponding to an internal quantum efficiency of around $1 \%$ which is comparable to other devices made previously in our laboratory [1] and in [18]. The inset of Figure 8 (b) shows the data on a log-log plot where the gradient yields the value of $2 / Z$ where $Z$ is used to identify the dominant recombination mechanism [19]. Depending on whether the value obtained for $Z$ is closer to 1,2 , or 3 , the dominant term is SRH, radiative, or Auger recombination. It can be seen that for currents between $30-100 \mathrm{~mA}$ the value of $Z$ is close to 2 indicating that radiative recombination dominates whereas at currents between $120-300 \mathrm{~mA}$ the value of $Z$ changes to 3 indicating the onset of Auger recombination at high carrier concentrations. We therefore attribute the low output power and the sublinear $L$-Icharacteristics measured from the device due to a combination of Auger recombination and hole leakage, although current crowding may also be a contributing factor at high injection.

Despite the successful growth and demonstration of EL from the InAsSbN QWs, the output power and efficiency at room temperature is lower than type II InAsSb/InAs QW LEDs made previously in our laboratory [6]. This 


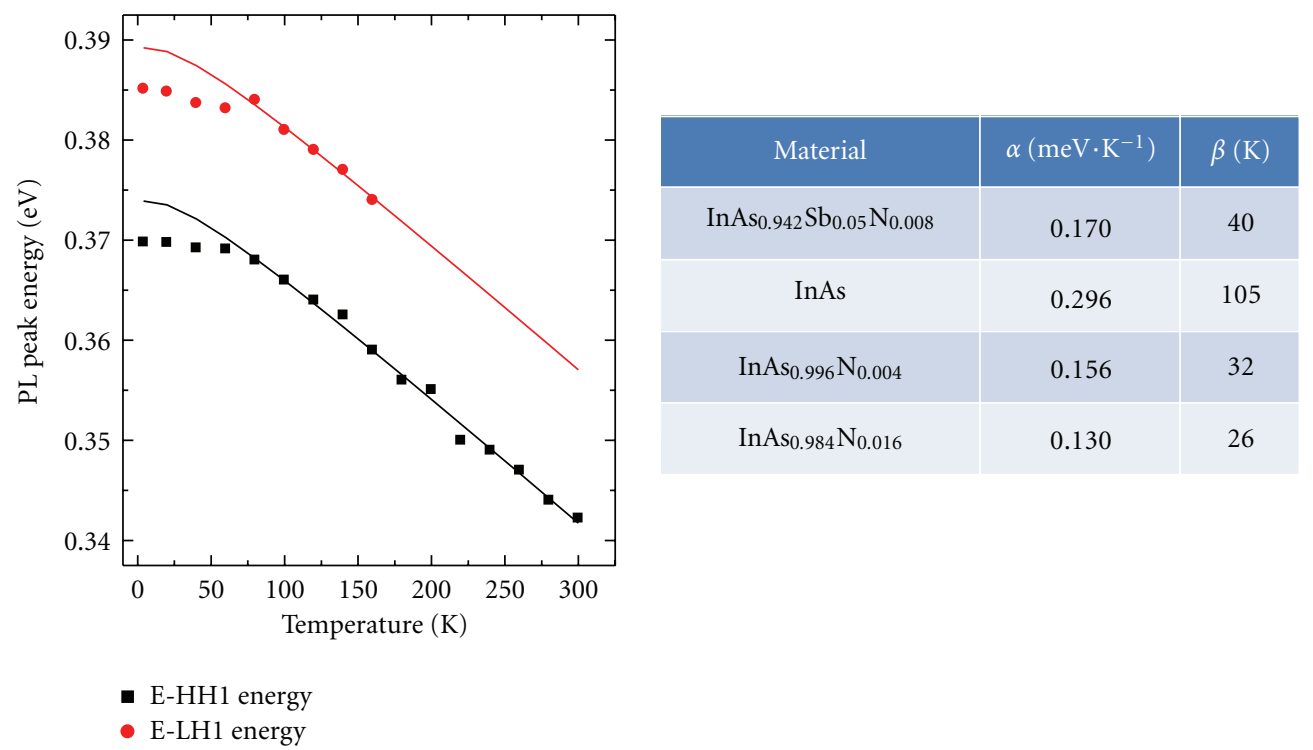

Figure 6: Temperature dependence of the EL emission peak energies. The continuous lines are calculated from the Varshni equation using $\alpha$ and $\beta$ as fitting parameters. The table lists the Varshni parameters obtained for $\operatorname{InAs}{ }_{0.942} \mathrm{Sb}_{0.05} \mathrm{~N}_{0.008}$ (this work), InAs [11], and InAsN [12].

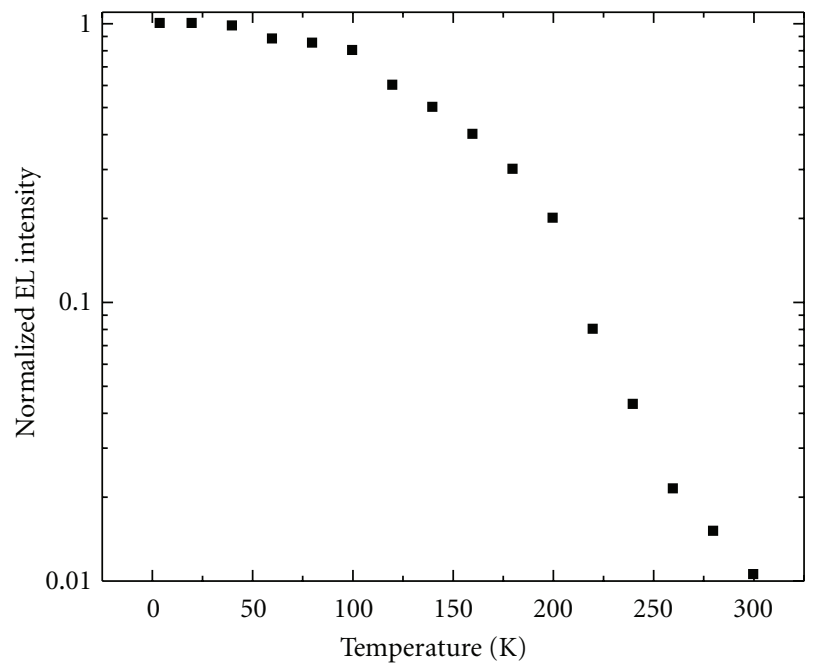

(a)

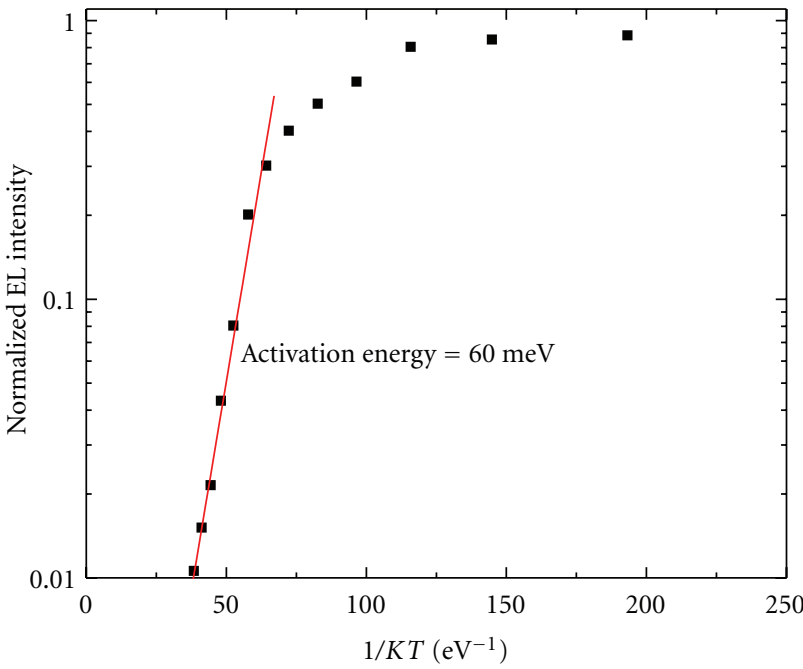

(b)

FIGURE 7: (a) Temperature dependence of the integrated normalized EL emission intensity for the e-hh1 transition. (b) Arrhenius plot from which an activation energy of $60 \mathrm{meV}$ is obtained from the gradient in the high temperature range.

is primarily due to the lower valence band offset (62 meV for InAsSb ${ }_{0.05} \mathrm{~N}_{0.008} /$ InAs compared to $86 \mathrm{meV}$ for InAsSb $\mathrm{I}_{0.08} / \mathrm{InAs} \mathrm{QWs}$ ) since less $\mathrm{Sb}$ was incorporated into the InAsSbN QWs such that holes can escape more easily. We also note that in the type II InAsSb/InAs QWs the emission is predominantly from the excited hh2 state at room temperature which increases the output power. No EL from the excited hole state is observed in the type I InAsSbN/InAs QWs due to selection rules and the shorter carrier lifetimes. Figure 9 plots the temperature dependence of the normalized integrated intensity for the individual transitions in both samples. It can be seen that in the temperature range $4-180 \mathrm{~K}$ the intensity from the e-hh1 transition in the InAsSbN/InAs wells is much higher and more stable than that from the InAsSb/InAs wells due to the larger radiative recombination rate. At higher temperatures the intensity drops dramatically (at a rate comparable to the e-hh1 transition in InAsSb/InAs) due to the loss of holes from the QW. In the InAsSb/InAs QWs, holes can escape from the ground state to the InAs barrier (as no emission is observed from InAs we assume they undergo Auger recombination) or be excited into the hh2 state. Here 


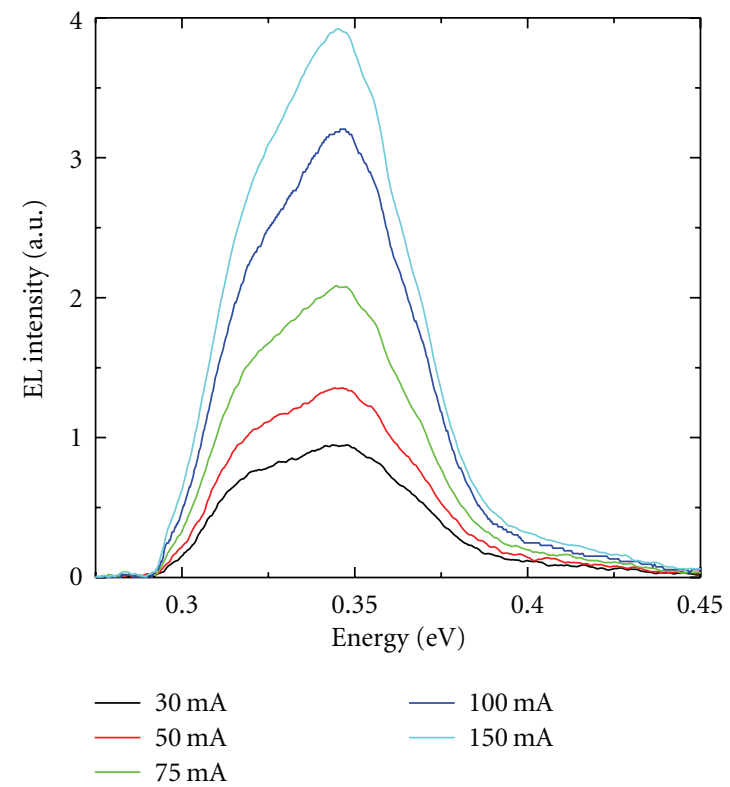

(a)

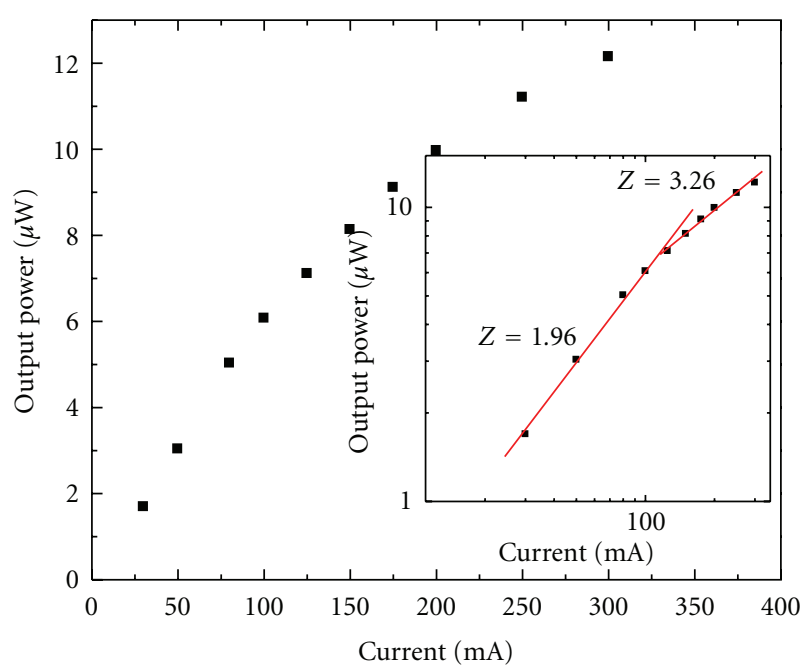

(b)

Figure 8: (a) Room temperature EL spectra measured at different drive currents using $1 \mathrm{kHz}$ and $50 \%$ duty cycle. (b) Measured output power at room temperature. The inset shows the data on a $\log -\log$ scale. The lines correspond to the gradients from which the values of $Z$ are extracted.

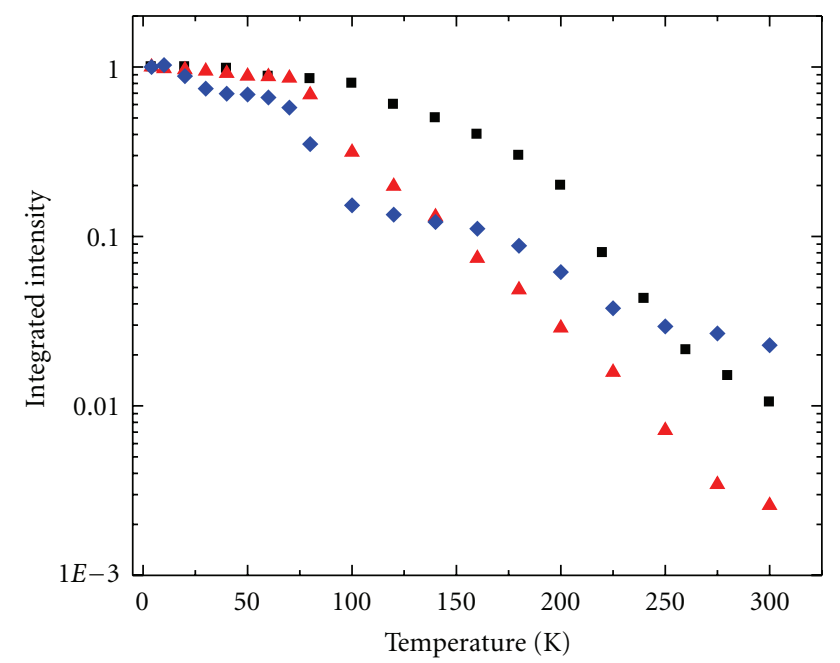

- InAsSbN e-hhl

- InAsSb e-hhl

$\rightarrow$ InAsSb e-hh2

Figure 9: Temperature dependence of the integrated emission intensity for the electron to confined heavy hole transitions in InAsSbN and InAsSb QW LEDs.

as observed they can recombine radiatively which improves the temperature quenching and output power of the LED.

To improve the performance of the device it is necessary to increase the hole confinement energy and heavy hole splitting in the wells and to confine electrons by increasing the conduction band offset. The former can be achieved by increasing the $\mathrm{Sb}$ content in the well and the latter by increasing the nitrogen content in the well. However, this is not straightforward since both effects work against each other. Increasing the nitrogen content reduces the compressive strain in the quantum well which reduces the valence band offset and the hh1-hh2 splitting. Increasing the Sb content increases the valence band offset but pushes the conduction band upwards which cannot be compensated easily by increasing the nitrogen content. These effects are illustrated in Figure 10 which plots the emission energy and band offsets for given amounts of $\mathrm{Sb}$ and $\mathrm{N}$. We estimate that using between $8-10 \% \mathrm{Sb}$ and $2.5 \% \mathrm{~N}$ in the well will give $82-104 \mathrm{meV}$ hole and $57-65 \mathrm{meV}$ electron confinement with an emission energy $256-270 \mathrm{meV}$ at $4 \mathrm{~K}$. Alternatively, the barrier height could be increased by using a wider band gap material such as InAsSbP or $\operatorname{InGaAs}(\mathrm{N})$ which will be the subject of our future work.

\section{Conclusion}

In summary, InAsSbN/InAs MQW LEDs have been grown by MBE which exhibit excellent structural quality and bright EL up to room temperature. The EL was identified as originating from a type I transition from electrons in the InAsSbN to confined hole states. Analysis of the temperature dependence of the EL suggests that thermally activated hole leakage is a limiting factor in these devices and that Auger recombination dominates at higher currents $(I>120 \mathrm{~mA})$. Further optimization of the QW band structure is anticipated to lead to mid-infrared devices which could be used in a variety of technologically important applications. 


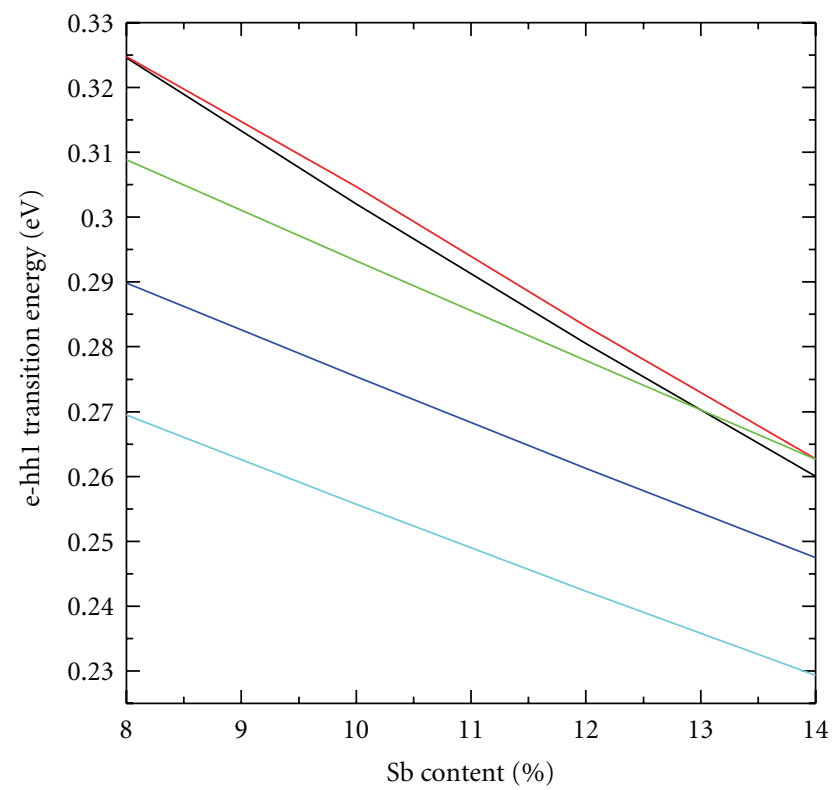

(a)

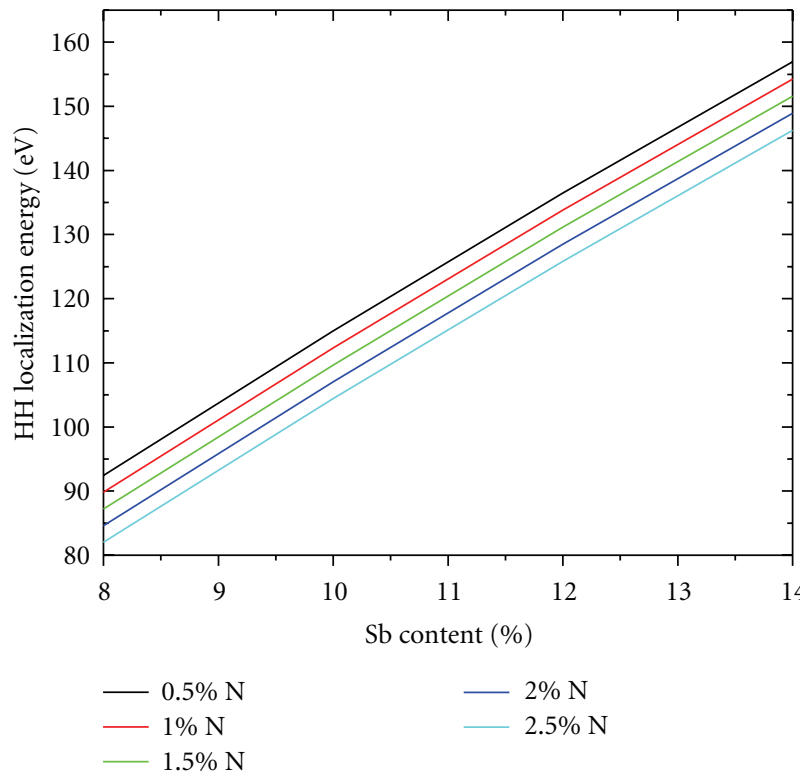

(b)

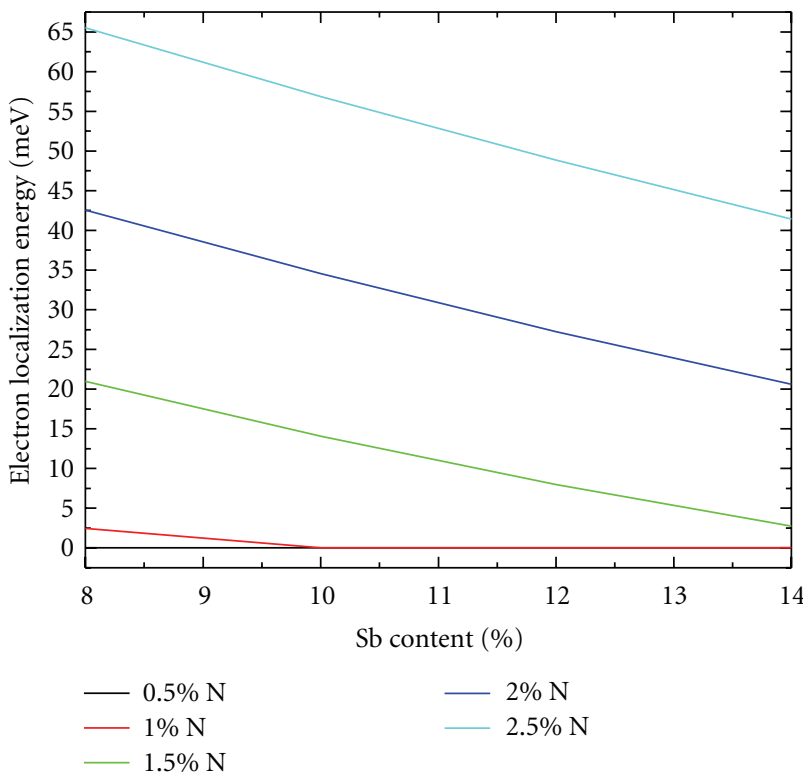

(c)

Figure 10: Calculated $4 \mathrm{~K}$ values of (a) e-hh1 transition energy (b) hh1 localization energy and (c) electron localization energy for different contents of $\mathrm{Sb}$ and $\mathrm{N}$ in a InAs $\mathrm{In}_{1-x-y} \mathrm{Sb}_{x} \mathrm{~N}_{y} / \mathrm{InAs} \mathrm{QW}$.

\section{Acknowledgments}

The authors would like to thank EPSRC for the award of a studentship to M. de la Mare and K. J. Cheetham. They also wish to thank J. Statter for his skilled technical support.

\section{References}

[1] P. J. Carrington, V. A. Solov'ev, Q. Zhuang, A. Krier, and S. V. Ivanov, "Room temperature midinfrared electroluminescence from InSb/InAs quantum dot light emitting diodes," Applied Physics Letters, vol. 93, no. 9, Article ID 091101, 2008.
[2] N. B. Cook and A. Krier, "Midinfrared electroluminescence from pentanary-quaternary heterojunction light-emitting diodes," Applied Physics Letters, vol. 95, no. 2, Article ID 021110, 2009.

[3] B. I. Mirza, G. R. Nash, S. J. Smith et al., "Recombination processes in midinfrared $\mathrm{Al}_{x} \mathrm{In}_{1-x} \mathrm{Sb}$ light-emitting diodes," Journal of Applied Physics, vol. 104, no. 6, Article ID 063113, 2008.

[4] S. Suchalkin, S. Jung, G. Kipshidze et al., "GaSb based light emitting diodes with strained InGaAsSb type i quantum well active regions," Applied Physics Letters, vol. 93, no. 8, Article ID 081107, 2008. 
[5] E. J. Koerperick, J. T. Olesberg, T. F. Boggess et al., "InAs/GaSb cascaded active region superlattice light emitting diodes for operation at $3.8 \mu \mathrm{m}$," Applied Physics Letters, vol. 92, no. 12, Article ID 121106, 2008.

[6] P. J. Carrington, Q. Zhuang, M. Yin, and A. Krier, “Temperature dependence of mid-infrared electroluminescence in type II InAsSb/InAs multi-quantum well light-emitting diodes," Semiconductor Science and Technology, vol. 24, no. 7, Article ID 075001, 2009.

[7] B. N. Murdin, M. Kamal-Saadi, A. Lindsay et al., "Auger recombination in long-wavelength infrared $\mathrm{InN}_{x} \mathrm{Sb}_{1-x}$ alloys," Applied Physics Letters, vol. 78, no. 11, pp. 1568-1570, 2001.

[8] Q. Zhuang, A. Godenir, A. Krier, G. Tsai, and H. H. Lin, "Molecular beam epitaxial growth of InAsN:Sb for midinfrared Optoelectronics," Applied Physics Letters, vol. 93, no. 12, Article ID 121903, 2008.

[9] M. de La Mare, P. J. Carrington, R. Wheatley et al., "Photoluminescence of InAs ${ }_{0.926} \mathrm{Sb}_{0.063} \mathrm{~N}_{0.011} /$ InAs multi-quantum wells in the mid-infrared spectral range," Journal of Physics D, vol. 43, no. 34, Article ID 345103, 2010.

[10] W. Y. Uen, S. M. Liao, C. T. Lin, and C. H. Wu, "Lowtemperature electrical characterizations of $\operatorname{InAs}_{1-x-y} \mathrm{Sb}_{y} \mathrm{P}_{x}$ photodiodes fabricated by liquid-phase epitaxy," Solid-State Electronics, vol. 46, no. 9, pp. 1405-1409, 2002.

[11] K. J. Cheetham, P. J. Carrington, N. B. Cook, and A. Krier, "Low bandgap GaInAsSbP pentanary thermophotovoltaic diodes," Solar Energy Materials and Solar Cells, vol. 95, no. 2, pp. 534-537, 2011.

[12] N. V. Zotova, N. D. Il'inskaya, S. A. Karandashev, B. A. Matveev, M. A. Remennyi, and N. M. Stus', "Flip-chip LEDs with deep mesa emitting at $4.2 \mu \mathrm{m}$," Semiconductors, vol. 40, no. 6, pp. 697-703, 2006.

[13] J. Hoffmann, T. Lehnert, D. Hoffmann, and H. Fouckhardt, "Advantages and disadvantages of sulfur passivation of InAs/GaSb superlattice waveguide photodiodes," Semiconductor Science and Technology, vol. 24, no. 6, article 065008, 2009.

[14] Y. P. Varshni, “Temperature dependence of the energy gap in semiconductors," Physica, vol. 34, no. 1, pp. 149-154, 1967.

[15] Z. M. Fang, K. Y. Ma, D. H. Jaw, R. M. Cohen, and G. B. Stringfellow, "Photoluminescence of InSb, InAs, and InAsSb grown by organometallic vapor phase epitaxy," Journal of Applied Physics, vol. 67, no. 11, pp. 7034-7039, 1990.

[16] A. M. R Godenir, Novel dilute nitride semiconductor materials for mid infrared applications, Ph.D. thesis, Lancaster University, Lancaster, UK, 2008.

[17] F. I. Lai, S. Y. Kuo, J. S. Wang et al., "Temperature-dependent optical properties of $\operatorname{In}_{0.34} \mathrm{Ga}_{0.66} \mathrm{As}_{1-x} \mathrm{~N}_{x} / \mathrm{GaAs}$ single quantum well with high nitrogen content for $1.55 \mu \mathrm{m}$ application grown by molecular beam epitaxy," Journal of Crystal Growth, vol. 291, no. 1, pp. 27-33, 2006.

[18] G. R. Nash, M. K. Haigh, H. R. Hardaway et al., "InSbAlInSb quantum-well light-emitting diodes," Applied Physics Letters, vol. 88, no. 5, Article ID 051107, pp. 1-3, 2006.

[19] S. J. Sweeney, A. F. Phillips, A. R. Adams, E. P. O'Reilly, and P. J. A. Thijs, "The effect of temperature dependent processes on the performance of $1.5-\mu \mathrm{m}$ compressively strained $\mathrm{InGaAs}(\mathrm{P})$ MQW semiconductor diode lasers," IEEE Photonics Technology Letters, vol. 10, no. 8, pp. 1076-1078, 1998. 

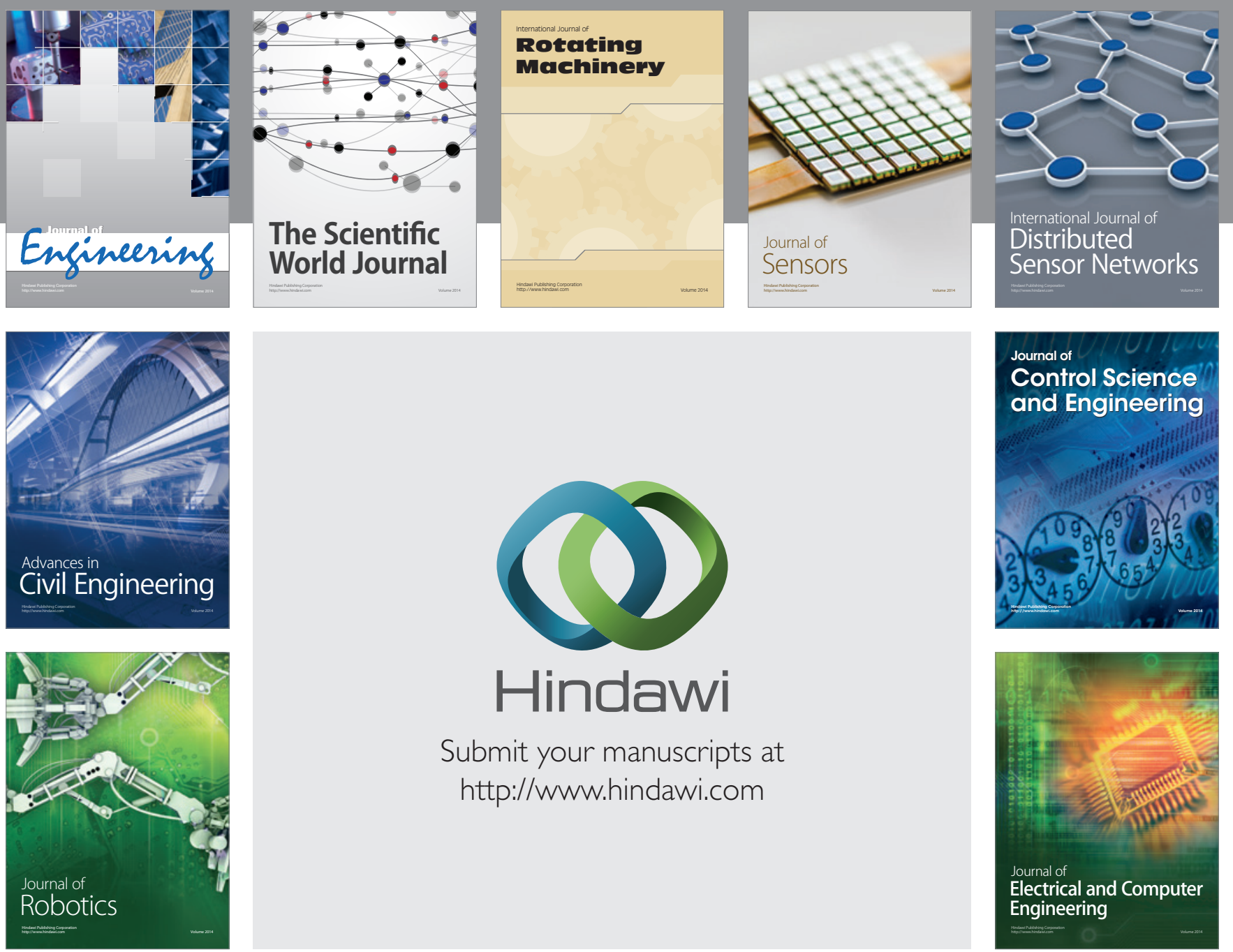

Submit your manuscripts at

http://www.hindawi.com
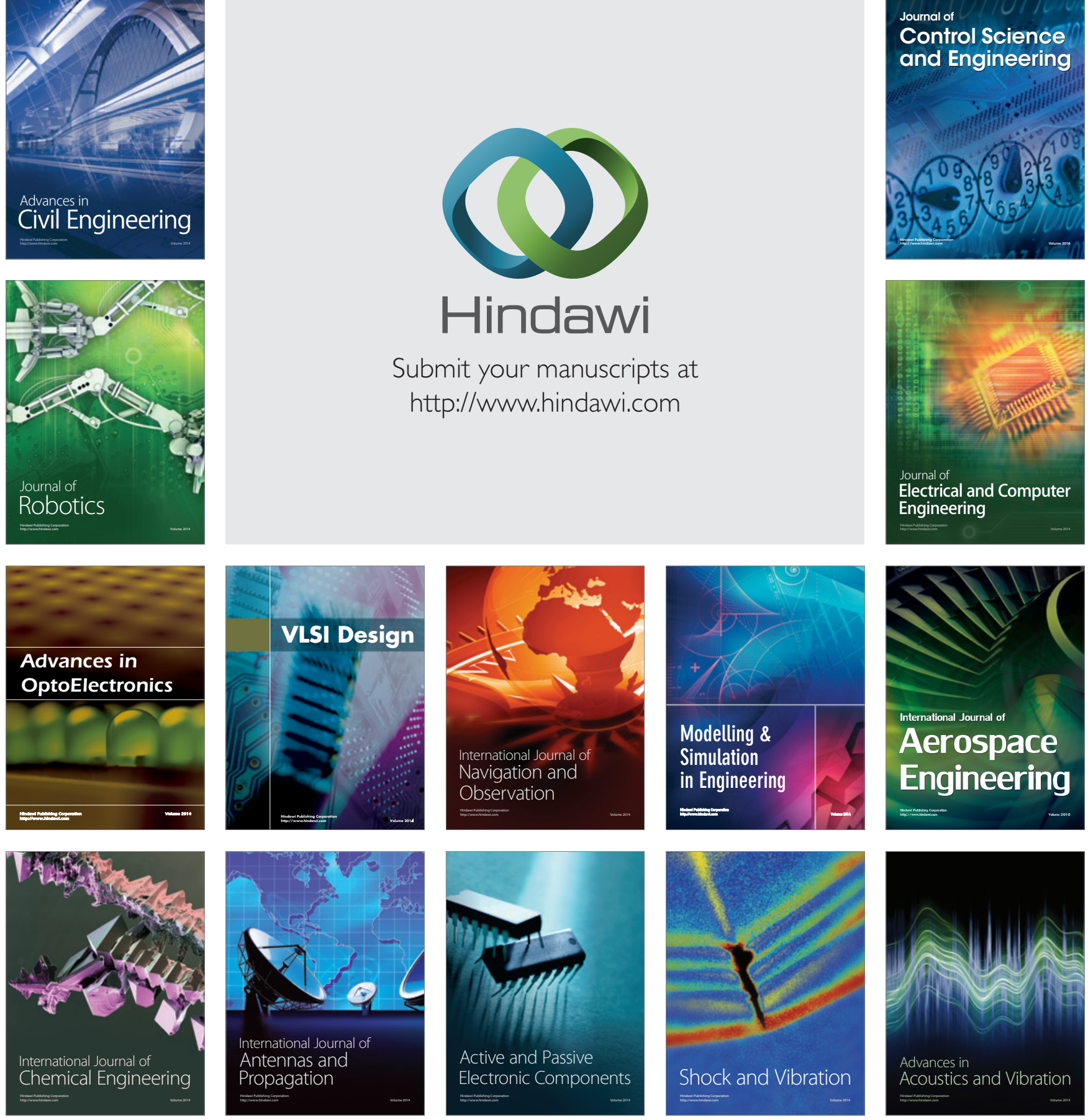